\title{
Lesión de Dieulafoy en estómago como causa de sangrado gastrointestinal alto: presentación de un caso
}

\author{
Jairo Alonso Sierra-Avendaño* \\ Fabián Andrés Mejía-Casadiegos ** \\ María Paula Pérez-Barón *** \\ Gabriel Eduardo Pérez-García****
}

\begin{abstract}
* Médico residente de I año de Patología. Departamento de Patología. Grupo de Patología Estructural, Funcional y Clínica de la Universidad Industrial de Santander (PAT-UIS). Facultad de Salud. Universidad Industrial de Santander. Bucaramanga. Colombia.

**Médico residente de III año de Patología. Departamento de Patología. Facultad de Salud. Universidad Industrial de Santander. Bucaramanga. Colombia.

***Estudiante de XI Semestre de Medicina. Escuela de Medicina. Facultad de Ciencias de la Salud. Universidad Autónoma de Bucaramanga. Colombia. ****Médico Patólogo. Coordinador de Posgrado de Patología. Departamento de Patología. Grupo de Patología Estructural, Funcional y Clínica de la Universidad Industrial de Santander (PAT-UIS). Facultad de Salud. Universidad Industrial de Santander. Bucaramanga. Colombia.

Correspondencia: Dr. Gabriel Eduardo Pérez García. Departamento de Patología. Universidad Industrial de Santander. Facultad de Salud. Carrera 32 No. 29-31. Bucaramanga. Colombia. Correo electrónico: geperezg@uis.edu.co. Teléfono: (+57) 3185320299.
\end{abstract}

Resumen

La lesión de Dieulafoy representa aproximadamente el $2 \%$ de todos los casos de hemorragia gastrointestinal superior. En la mayoría de casos esta anomalía se ubica en el estómago, principalmente en la curvatura menor. Se presenta el caso de un adulto joven con hematemesis y signos de hipovolemia. Durante la esofagogastroduodenoscopia se evidenció un coágulo adherido en el cuerpo gástrico a nivel de la curvatura mayor, sugestivo de lesión de Dieulafoy. Inicialmente se realizó manejo médico sin mejoría significativa, por lo que se realizó laparotomía exploratoria con resección de la malformación vascular, finalmente con el informe histopatológico se confirmó el diagnóstico. A pesar de ser una causa infrecuente de hemorragia gastrointestinal superior, la lesión de Dieulafoy debe considerarse entre los diagnósticos diferenciales, especialmente si la hemorragia persiste aún con manejo médico. MÉD.UIS.2019;32(1):27-31.

Palabras clave: Malformaciones vasculares. Hemorragia gastrointestinal. Enfermedades gastrointestinales. Patología quirúrgica. Laparotomía.

\section{Dieulafoy's lesion in stomach as cause of upper gastrointestinal bleeding: case report}

Abstract

Dieulafoy's lesion represents approximately $2 \%$ of all causes of upper gastrointestinal bleeding. In most cases, this malformation is located in the stomach, mainly in the lesser curvature. We present the case of a young adult with hematemesis and signs of hypovolaemia. During the esophagogastroduodenoscopy, a clot adhering to the gastric body was seen at the level of the greater curvature, suggestive of Dieulafoy's lesion. Initially, medical management was performed without significant improvement, so an exploratory laparotomy was performed with resection of the vascular malformation, the histopathological report confirmed the diagnosis. Despite being an uncommon cause of upper gastrointestinal bleeding, the Dieulafoy lesion must be considered among the differential diagnoses, especially if the hemorrhage persists even with medical management. MÉD.UIS.2019;32(1):27-31.

Keywords: Vascular malformations. Gastrointestinal hemorrhage. Gastrointestinal diseases. Surgical pathology. Laparotomy.

Artículo recibido el 11 de julio del 2018 y aceptado para publicación el 1 de marzo del 2019. 
¿Cómo citar este artículo?: Sierra-Avendaño JA, Mejía-Casadiegos FA, Pérez-Barón MP, PérezGarcía GE. Lesión de Dieulafoy en estómago como causa de sangrado gastrointestinal alto: presentación de un caso. MÉD.UIS.2019;32(1):27-31. doi: 10.18273/revmed.v32n1-2019004

\section{Introducción}

La hemorragia gastrointestinal se define con relación a la localización con respecto al ligamento de Treitz, se clasifica como superior, al estar proximal o inferior, cuando se encuentra distal al ligamento'. La hemorragia gastrointestinal superior es una emergencia médica frecuente y su incidencia estimada es de 100 por cada 100000 adultos $^{2}$. Las úlceras pépticas son la causa más frecuente de hemorragia gastrointestinal superior no variceal correspondiendo del 31 al $67 \%$, mientras que existen otras menos comunes como las lesiones vasculares que incluyen la angiodisplasia, la lesión de Dieulafoy y la ectasia vascular antral gástrica que producen entre el 2 al $8 \%$ de los episodios de sangrado superior ${ }^{3}$.

La lesión de Dieulafoy fue descrita originalmente por Gallard en 1884 y catorce años después fue denominada como "exulceración simple" por el cirujano francés Georges Dieulafoy4. Esta lesión también llamada "arteria de calibre persistente", consiste en arterias submucosas aberrantes, dilatadas y de trayecto tortuoso que erosionan la mucosa, con ruptura espontánea y sangrado gastrointestinal severo, relacionadas con tasas de mortalidad de más del 80\%,5.6 La lesión de Dieulafoy ha sido reportada en el esófago, duodeno, intestino delgado y colon, pero su ubicación predilecta es la cavidad gástrica en el $71 \%$ de los casos 7 . Esta patología es una causa infrecuente de hemorragia gastrointestinal superior con prevalencia de apenas el $1-2 \%$ y representa hasta el $0,1 \%$ de las hemorragias agudas ${ }^{8}$.

La patogenia de esta lesión es desconocida, no se han descrito mutaciones genéticas asociadas, sin embargo, existen algunos factores que se han relacionado a su presentación tales como el envejecimiento, el género masculino (relación 2:1) y comorbilidades como enfermedades cardiovasculares (enfermedad coronaria, falla cardíaca, valvulopatías e hipertensión arterial), diabetes y enfermedad renal crónica ${ }^{8}$. La lesión permanece asintomática hasta que la erosión de la mucosa expone la arteria subyacente provocando el sangrado. Factores como el consumo de alcohol, antiinflamatorios no esteroides y anticoagulantes se han asociado con los episodios de ruptura?.
Clínicamente se caracteriza por cuadros repetitivos o intermitentes de dolor abdominal, hematemesis, melenas o sangrado profuso e indoloro que requieren transfusión con hemoderivados debido a los signos de inestabilidad hemodinámica (hipotensión, taquicardia, ortostatismo y azoemia prerrenal) ${ }^{10,11}$.

La prueba diagnóstica de elección es la esofagogastroduodenoscopia, sin embargo, este método puede fracasar frente a lesiones pequeñas, mucosa de aspecto normal, patrón de sangrado intermitente o excesiva hemorragia que dificulte la inspección, por lo que la tomografía computarizada con contraste o la angiografía toman importancia como alternativas para realizar el diagnóstico ${ }^{6}$.

El tratamiento primario de las lesiones de Dieulafoy suele ser mediante métodos endoscópicos que incluyen electrocoagulación, escleroterapia e inyección local de epinefrina y el uso de bandas mecánicas, sin que alguna modalidad haya demostrado superioridad y por este motivo se recomiende el uso dependiendo de la preferencia y experiencia del endoscopista ${ }^{12,13}$. La angiografía con embolización y la cirugía se reservan para los casos donde no es posible identificar la lesión mediante la endoscopia ${ }^{14}$.

A continuación, se reporta el caso de un adulto joven masculino que cursó con episodios recurrentes de hemorragia gastrointestinal superior sin diagnóstico previo establecido. El objetivo del presente trabajo es describir la lesión de Dieulafoy desde un abordaje clínico, histopatológico y como un diagnóstico desafiante en casos de sangrado gastrointestinal recurrente.

\section{Presentación de caso}

Masculino de 29 años remitido del área rural de Santander, Colombia, quien consultó al servicio de urgencias del Hospital Universitario de Santander por tres episodios de hematemesis y tres de melenas en las últimas ocho horas asociado a dolor abdominal, astenia, adinamia y somnolencia. Como antecedentes importantes se documentó retardo mental moderado, tabaquismo y dos episodios de 
hemorragia digestiva superior, el último en 2014, sin etiología definida, que requirieron hospitalización y transfusión de hemoderivados. Negó consumo crónico de alcohol y antiinflamatorios no esteroides. Al examen físico se evidenció regular estado general, con signos de hipovolemia, alteración del estado de conciencia, tendencia a la hipotensión y taquicardia, palidez mucocutánea, llenado capilar lento y leve dolor abdominal a la palpación sin signos de irritación peritoneal.

Se diagnosticó hemorragia gastrointestinal superior de etiología a definir. Fue manejado en el momento del ingreso con oxígeno a tres litros por minuto con cánula nasal, bolo endovenoso de $1000 \mathrm{~mL}$ de lactato de Ringer y luego de $100 \mathrm{~mL} / \mathrm{h}$, omeprazol de $40 \mathrm{mg}$ endovenoso cada 12 horas y metoclopramida de 10 $\mathrm{mg}$ endovenoso cada 8 horas. Debido a los signos de hipovolemia se realizaron exámenes paraclínicos iniciales que reportaron hemoglobina de $7.2 \mathrm{~g} / \mathrm{dL}$, hematocrito de $21.4 \%$ VCM $88.8 \mathrm{fL}$, HCM de 29.9 $\mathrm{pg}$, recuento leucocitario de 6430 , recuento de plaquetas de 139000 , nitrógeno uréico en sangre $40.87 \mathrm{mg} / \mathrm{dL}$ y creatinina sérica de $0.76 \mathrm{mg} / \mathrm{dL}$. Se ordenó transfusión de tres unidades de glóbulos rojos empaquetados y una esofagogastroduodenoscopia. Adicionalmente por el hallazgo de trombocitopenia se decidió descartar hepatopatía con estudios complementarios sin alteraciones significativas en los valores de transaminasa glutámico oxalacética 20.1 UL, transaminasa glutámico pirúvica de 17.5 UL, fosfatasa alcalina de $31 \mathrm{U} / \mathrm{L}$, tiempo de protrombina de 11.2 segundos y tiempo de tromboplastina de 22.6 segundos, acompañado de ecografía hepatobiliar sin alteraciones en el parénquima hepático ni en la vía biliar intra ni extrahepática.

A los dos días del ingreso se realizó la esofagogastroduodenoscopia bajo sedación reportando vaso visible pulsátil de $4 \mathrm{~mm}$ en el cuerpo gástrico sobre la curvatura mayor, con presencia de coágulo adherido, en el momento sin sangrado, con resto de mucosa gástrica, esofágica y duodenal de aspecto usual, por lo cual se considera lesión de Dieulafoy en cuerpo gástrico y se recomienda manejo quirúrgico inmediato con resección de lesión y posibilidad de gastrectomía subtotal dado que no se contaba con manejo endoscópico en la institución.

Durante la estancia hospitalaria entre el tercer y quinto día el paciente se mantuvo con estabilidad hemodinámica, sin nuevos episodios de hematemesis, melenas, dolor abdominal ni cambios significativos en los niveles de hemoglobina, hematocrito, recuento plaquetario ni alteraciones hidroelectrolíticas.

Al sexto día de hospitalización se realizaron laparotomía exploratoria, gastrotomía y resección de lesión de Dieulafoy, sin complicaciones intra ni posoperatorias. Dentro de los hallazgos quirúrgicos se reportó lesión erosionada de bordes irregulares de aproximadamente $8 \mathrm{~mm}$ con vaso sangrante. Se da egreso hospitalario al séptimo día de postoperatorio tras tolerar la vía oral, con recomendaciones e instrucciones de consultar al servicio de urgencias en caso de presentar fiebre, dolor abdominal, signos de infección de la herida quirúrgica o nuevos episodios de hematemesis y melenas.

Los hallazgos histopatológicos mostraron pared gástrica oxíntica ulcerada en la que se reconoció edema, congestión vascular, áreas de hemorragia, infiltrado inflamatorio mixto de predominio mononuclear, depósitos de fibrina, detritus celulares y fibrosis focal asociados a gran vaso arterial en la submucosa, rodeado por fibrosis. No se evidenciaron bacilos de tipo Helicobacter, displasia o malignidad en el epitelio glandular. Los hallazgos fueron consistentes con malformación vascular sangrante de tipo lesión Dieulafoy en pared gástrica (Ver Figura 1).

En la consulta de control por gastroenterología, ocho días después del alta hospitalario no se evidenciaron signos de sangrado. El paciente no ha consultado al servicio de urgencias del Hospital Universitario de Santander por nuevos episodios de sangrado gastrointestinal.

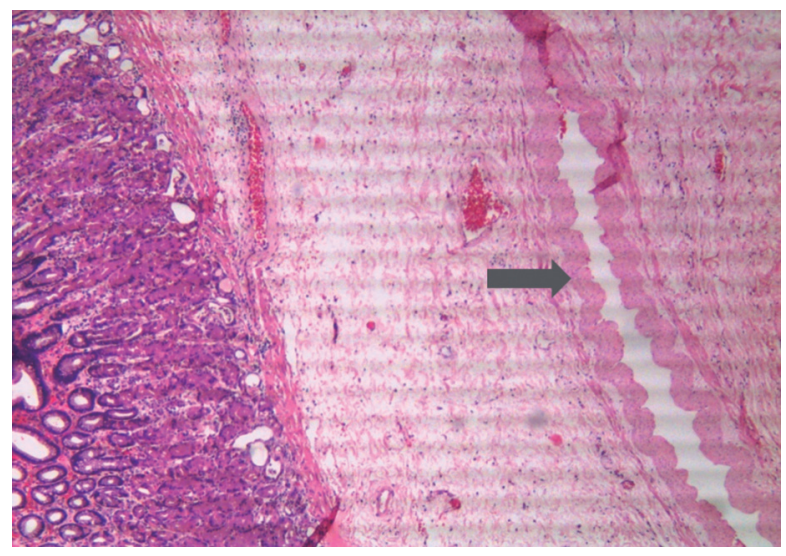

Figura 1. Histopatología de la pared gástrica. Pared gástrica oxíntica con gran vaso arterial tortuoso y dilatado (flecha gris) ubicado en la submucosa, rodeado por fibrosis, subyacente al área erosionada. Tinción de hematoxilina y eosina (H\&E); magnificación $\times 12.5$

Fuente: Departamento de Patología, Universidad Industrial de Santander. 


\section{Discusión}

Los pacientes con lesiones de Dieulafoy frecuentemente se presentan con melenas, hematemesis, hematoquecia, anemia, o una combinación de éstas, siendo las melenas las más comunes $^{15}$. La hemorragia aguda masiva, a veces recurrente, puede considerarse el sello durante la presentación clínica de estas lesiones, tal como se evidenció en el presente caso. De acuerdo a lo descrito por Baxter, hasta el 44\% pueden cursar con melenas, $30 \%$ con hematemesis, $18 \%$ con una combinación de las dos y $6 \%$ con hematoquecia ${ }^{16}$. El paciente reportado hace parte del grupo con presentación mixta de hematemesis y melenas.

No existen estudios retrospectivos con muestras significativas ni aleatorizados que describan adecuadamente los factores de riesgo cardiovasculares y los factores pronóstico. Kamal y colaboradores en el Hospital Cabell Huntington de Virginia mostraron en un estudio retrospectivo un grupo de 36 pacientes con diagnóstico previo de lesión de Dieulafoy; 24 de ellos eran hipertensos con, por lo menos, un episodio de hemorragia gastrointestinal superior causada por la ruptura de la lesión, encontrando que todos estos pacientes son adultos mayores y con niveles significativamente bajos de hemoglobina (7,9 $\mathrm{g} / \mathrm{dL}+/-2,4$ entre los pacientes hipertensos y 11,1 g/dL+/- 3,4 entre los pacientes normotensos). Este paciente no cumple con los factores de riesgo en dicho estudio, puesto que se trata de un paciente joven y normotenso, lo cual apunta a un mejor pronóstico. Posterior al control del sangrado, los pacientes con lesiones de Dieulafoy tienden a reducir significativamente sus tasas de mortalidad ${ }^{17}$. Probablemente, los factores cardiovasculares puedan contribuir con el remodelamiento de estas lesiones y el riesgo de resangrado.

Adicionalmente, Shin y colaboradores estudiaron una población de 42 pacientes con diagnóstico de Lesión de Dieulafoy y 132 pacientes con hemorragia gastrointestinal superior por otra causa, encontrando como factor de riesgo asociado a la formación de lesión de Dieulafoy el consumo de agentes antiplaquetarios $(p=0.022)$ y alcohol $(p=0.001)$. No hubo relación estadística con el consumo de antiinflamatorios no esteroides $(p=0.663)$ y tabaquismo $(p=0.101)^{18}$. Estos factores difieren del caso aquí presentado ya que en este paciente se encontró el antecedente de tabaquismo y no se reportó consumo de antiinflamatorios no esteroides, alcohol ni agentes antiplaquetarios, en concordancia con lo descrito por otros autores ${ }^{7,14}$.

En un estudio retrospectivo se evaluaron pacientes con esta patología para definir los factores predictores de resangrado, encontrando que la enfermedad renal y la infección son predictores mayores de resangrado y mortalidad, por lo que las enfermedades subyacentes deben ser tomadas en cuenta como un punto importante para mejorar el pronóstico de los pacientes ${ }^{19}$.

En cuanto a las pruebas diagnósticas la esofagogastroduodenoscopia inicial es eficaz en el diagnóstico de hasta el 70\% de los pacientes, sin embargo, se puede requerir varias endoscopias; incluso $6 \%$ de los casos son necesarios tres o más endoscopias para establecer el diagnóstico ${ }^{8,10}$. Lo anterior fue evidenciado en el caso presentado con tres esofagogastroduodenoscopia realizadas durante la enfermedad actual, lo que reivindica este diagnóstico como desafiante en el contexto de una hemorragia gastrointestinal superior recurrente sin etiología establecida. Más del 70\% de los hallazgos anatomopatológicos y endoscópicos reportados de lesión de Dieulafoy se ubican en el estómago, específicamente hacia la curvatura menor a $6 \mathrm{~cm}$ de la unión gastroesofágica ${ }^{10,20,21}$, sin embargo, la ubicación de la lesión en este paciente se encuentra en la curvatura mayor. Del caso expuesto se debe reconocer como limitación importante que no se presentan las imágenes endoscópicas de la lesión.

Por tanto, la confirmación diagnóstica es netamente histopatológica para descartar diagnósticos diferenciales como la angiodisplasia y la úlcera péptica sangrante. Por lo general las angiodisplasias se acompañan de arterias y venas submucosas anormales, mientras que en la lesión Dieulafoy se observa una única arteria submucosa anormalmente superficial, de gran calibre y erosión de la mucosa circundante como se evidenció en el presente caso ${ }^{19}$.

\section{Conclusiones}

La lesión de Dieulafoy es de las causas menos frecuentes de hemorragia gastrointestinal superior, sin embargo, es importante reconocerla y sospecharla en pacientes de cualquier edad que se presenten con episodios de sangrado digestivo recurrentes. Los métodos endoscópicos constituyen el pilar diagnóstico y terapéutico, su confirmación se 
obtiene a través de histopatología descartando otras posibles causas.

\section{Consideraciones éticas}

Se contó con el correspondiente consentimiento informado para el reporte del caso, así como también para el uso de los datos y hallazgos consignados en la historia clínica del paciente.

\section{Conflictos de intereses}

Ninguno.

\section{Referencias bibliográficas}

1. Feinman M, Haut ER. Upper gastrointestinal bleeding. Surg Clin North Am 2014; 94(1):43-53

2. Loperfido S, Baldo V, Piovesana E, Bellina L, Rossi K, Groppo M, et al. Changing trends in acute upper-GI bleeding: a populationbased study. Gastrointest Endosc. 2009; 70(2):212-24.

3. Samuel R, Bilal M, Tayyem O, Guturu P. Evaluation and management of Non-variceal upper gastrointestinal bleeding. Dis Mon. 2018; 64(7):333-43.

4. Lee YT, Walmsley RS, Leong RW, Sung JJY. Dieulafoy's lesion. Gastrointest Endosc. 2003;58(2):236-43.

5. al-Mishlab T, Amin AM, Ellul JP. Dieulafoy's lesion: an obscure cause of GI bleeding. J R Coll Surg Edinb. 1999:44(4):222-25.

6. Khalid S, Abbass A, Do T, Malhotra D, Albors-Mora M. The hidden culprit in a massive episode of hematemesis: a Dieulafoy's lesion. Cureus. 2016;8(10):e824.

7. Nguyen DC, Jackson CS. The Dieulafoy's lesion: an update on evaluation, diagnosis, and management. J Clin Gastroenterol 2015; 49(7):541-49.

8. Rojas A, Carvajal GD, Prieto RG, Aponte DM. Lesión de Dieulafoy de duodeno: hallazgo inusual. Rev Col Gastroenterol. 2016; 31(3):292-96.

9. Jain R, Chetty R. Dieulafoy disease of the colon. Arch Pathol Lab Med. 2009;133(11):1865-67.

10. Nojkov B, Cappell MS. Gastrointestinal bleeding from Dieulafoy's lesion: Clinical presentation, endoscopic findings, and endoscopic therapy. World J Gastrointest Endosc. 2015; 7(4):295-307.

11. Park J, Ha H, Kim M. Death due to gastrointestinal hemorrhage associated with Dieulafoy's lesion. Korean J Leg Med. 2014; 38(3):113-15.

12. Khan R, Mahmad A, Gobrial M, Onwochei F, Shah K. The diagnostic dilemma of Dieulafoy's lesion. Gastroenterology Res. 2015; 8(3-4):201-06.

13. Ding YJ, Zhao L, Liu J, Luo HS. Clinical and endoscopic analysis of gastric Dieulafoy's lesion. World J Gastroenterol. 2010; 16(5):63135 .

14. Jammal M, Khodorkovsky B, Raden M, Hahn B. Adult female with hematemesis-Dieulafoy lesion. Am J Emerg Med 2013; 31(5):889.

15. Saleh R, Lucerna A, Espinosa J, Scali V. Dieulafoy lesion: the little known sleeping giant of gastrointestinal bleeds. Am J Emerg Med. 2016; 34(12):2464.e3-2464.e5.

16. Baxter M, Aly EH. Dieulafoy's lesion: current trends in diagnosis and management. Ann R Coll Surg Engl. 2010; 92(7):548-54.

17. Kamal M, Santhanam P, Rayyan YM. Hypertension and clinical outcomes of patients with gastrointestinal submucosal vascular (Dieulafoy) lesional hemorrhage. J Clin Hypertens. 2016;18(7):710.

18. Shin HJ, Ju JS, Kim KD, Kim SW, Kang SH, Kang SH, et al. Risk factors for Dieulafoy lesions in the upper gastrointestinal tract. Clin Endosc. 2015; 48(3):228-33.

19. Park SH, Lee DH, Park CH, Jeon J, Lee HJ, Lim SU, et al. Predictors of rebleeding in upper gastrointestinal Dieulafoy lesions. Clin Endosc. 2015; 48(5):385-91.

20. Inayat F, Ullah W, Hussain Q, Hurairah A. Dieulafoy's lesion of the oesophagus: a case series and literature review. BMJ Case Rep. 2017. pii: bcr2016218100.

21. Fujimaru T, Akahoshi K, Matsuzaka H, Sumita Y, Kubokawa M. Bleeding rectal Dieulafoy's lesion. Gastrointest Endosc. 2003; 57(7):9-22. 